\title{
L a sutura compresiva del útero en cesárea con atonía uterina
}

\author{
PERCY PACORA 1,2,3, ALVARO SANTIVAÑEZ1,2,3, MÁXIMO AYALA ${ }^{1,2,3}$ \\ ${ }^{1}$ Departamento de Gineco-Obstetricia, Facultad de Medicina - UNM SM. \\ ${ }^{2}$ Hospital Madre-Niño "San Bartolomé". ${ }^{3}$ nnstituto de Patología, Facultad de Medicina - U NM SM
}

\begin{abstract}
Resumen
O bjetivo: Determinar si el empleo de la sutura compresiva del útero (técnica de B-L ynch) durante la cesárea en casos de atonía uterina disminuye la pérdida sanguínea y evita la histerectomía posparto. M aterial y M étodos: Estudio retrospectivo de las cesáreas que cursaron con atonía uterina durante la intervención, desde el 1 de enero de 1990 al 31 de diciembre de 2003, en el H ospital San Bartolomé de Lima. El método estándar de manejo de la atonía uterina en la cesárea consiste en el masaje uterino, el empleo de agentes uterotónicos (ocitocina, prostaglandinas), ligadura de arterias uterinas/ hipogástricas y la histerectomía posparto. Las variables maternas y perinatales obtenidas de las historias clínicas fueron ingresadas a una base de datos computarizado (SPSS versión 10). Resultados: Se identificó 46 casos: en 30 se empleó la técnica B-L ynch y en 16 el método estándar. No hubo diferencias en las características maternas entre ambos grupos. El grupo de B-Lynch presentó subjetivamente menor perdida sanguínea, recibió menor número de unidades de sangre transfundida y presentó significativo menor riesgo de histerectomía posparto comparado con el método estándar (promedio $\pm \mathrm{DE}: 1144,4 \pm 311,7 \mathrm{~mL}$ vs $1666,9 \pm 794,4 \mathrm{~mL}, \mathrm{p}<0,05 ; 0,36 \pm 0,66$ vs $2,90 \pm 3,64$, $p<0,05$; y OR 0,08, IC $95 \%$ 0,01-0,45, $p<0,01$, respectivamente). Conclusiones: La sutura compresiva del útero durante la cesárea en la atonía uterina parece reducir el sangrado vaginal, la transfusión sanguínea y el empleo de la histerectomía comparado con el método estándar.
\end{abstract}

Palabras clave: Hemorragia posparto; cesárea; suturas; útero.

\section{Uterine compression suture in uterine atony during cesarean section Abstract}

O bjetive: To ascertain whether the use of uterine compression suture ( $B-L y n c h$ technique) during a cesarean section with uterine atony decreases the amount of blood loss and prevents postpartum hysterectomy. Material and Methods: Retrospective study of all the cesarean section deliveries complicated by uterine atony from J anuary 1, 1990 through December 31, 2003 at San Bartolome Hospital in Lima. The standard management of uterine atony included uterine massage, use of uterotonic agents (ocytocin, prostaglandins), uterine/hypogastric artery ligature and, finally, postpartum hysterectomy. $M$ aternal and Infant clinical charts were reviewed and variables were entered into a computer system analysis (SPSS version 10). Results: Forty six cases were identified: B-L ynch technique was used in 30 cases and the standard method in 16 cases. There was no difference in maternal characteristic between both groups. B-L ynch technique group presented subjective lower amount of blood loss, received lower units of blood transfusion and had significant lower risk of postpartum hysterectomy as compared to the standard method group (mean \pm SD: $1144,4 \pm 311,7$

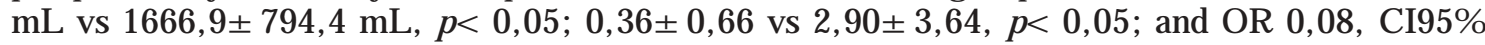
$0,01-0,45, p<0,01$, respectively). Conclusions: U terine compression sutures during a cesarean section with uterine atony may reduce the amount of blood loss, the number of blood transfusions and the use of hysterectomy as compared to the standard method.

Key words: Postpartum hemorrhage; cesarean section; sutures; uterus.

\section{INTRODUCCIÓN}

Se estima que más de 125,000 mujeres fallecen de hemorragia postparto en el mundo anualmente ${ }^{1}$ ). La hemorragia puerperal en el
Perú constituye la principal causa de muerte materna $\left(^{2-4}\right)$. La hemorragia puerperal se presenta en $1 \%(460 / 45,561)$ del total de partos atendidos en el Hospital $\mathrm{N}$ acional Docente $\mathrm{M}$ adre Niño San Bartolomé $\left(^{5}\right)$. 
El manejo tradicional de esta condición incluye el empleo de ocitócicos, tales como el Syntocinon (ocitocina sintética), ergometrina y las prostaglandinas, seguida de la ligadura de las arterias uterinas o las arterias iliacas internas y, como último recurso, se acude a la histerectomía. En 1997, B-L ynch y colaboradores reportaron un nuevo abordaje de tratamiento quirúrgico de la atonía uterina $\left({ }^{6}\right)$. Esta técnica consiste en el pasaje de una sutura del útero con una sutura continua para aplicar una compresión sostenida de las paredes del útero $\left({ }^{7}\right)$. E ste procedimiento es mucho más rápido y simple que efectuar una histerectomía o la ligadura de la arteria ilíaca interna y el reporte de una serie de casos en diversas partes del mundo sugiere que esta técnica es muy efectiva ( $\left.{ }^{8-14}\right)$.

Es necesario extraer el útero de la cavidad abdominal luego de la extracción de la placenta. Una vez confirmada la flacidez del útero y confirmado que no hay restos de placenta en la cavidad uterina, nos encontramos ante una atonía uterina y se procede a realizar la sutura compresiva del útero. La técnica consiste en el pasaje de una sutura continua con cátgut cromico No. 2 en la cara anterior y posterior del útero, empleando aguja curva con punta redonda de 6 $\mathrm{cm}$ de diámetro. U na vez terminado el pasaje de la sutura, según se observa en la Figura 1 , el cirujano jala los extremos de la sutura mientras el ayudante comprime las paredes del útero en sentido vertical y antero-posterior en forma sostenida, para poder amarrar los extremos del hilo, ejerciendo una compresión sostenida entre las paredes del útero. A I final, el útero tiene el aspecto de un tamal. A ntes de colocar el útero en la cavidad abdominal, el cirujano debe cerciorarse que no hay sangrado en los puntos de salida del cátgut crómico. En caso de sangrado, se utiliza puntos hemostáticos en " $U$ " con cátgut crómico 0 y aguja de $3 \mathrm{~cm}$ de diámetro y/o se realiza compresión manual con gasa durante 3 minutos. L os hilos del cátgut se absorben espontáneamente al cabo de 14 días, por lo que no requieren ser removidos.

La sutura compresiva del útero realiza una tensión sostenida en las fibras musculares en sentido vertical y antero-posterior. Esto permite la adhesión de la pared anterior y posterior del útero y el «acortamiento» de las paredes del útero en sentido vertical, de tal forma que no permite la formación del espacio virtual del endometrio, impidiendo la colección de sangre y realizaría el cierre "compresivo" de los vasos de las arterias espirales. Puede realizarse también la sutura compresiva empleando puntos en $U$, como se ve en la Figura 2, que son variantes de la técnica y emplear tantos puntos en $U$ como sea necesario, empleando siempre cátgut crómico grueso No. 2.

Desde el año 2000, esta técnica ha sido empleada por nosotros y la hemos enseñado en diversos maternidades de L ima y provincias (15,16). EI propósito de esta investigación es determinar si el empleo de la sutura compresiva del útero (técnica de B-L ynch) durante la cesárea disminuye la pérdida sanguínea y si evita la histerectomía posparto, comparado con el método estándar.

\section{MATERIAL Y MÉTODOS}

Se realizó un estudio retrospectivo de los partos por cesárea que cursaron con atonía uterina durante la operación, desde el 1 de enero 1990 al 31 de diciembre 2003, en el Hospital Madre-Niño "San Bartolomé" de Lima. El método estándar de manejo de la atonía uterina en la cesárea consiste en el masaje uterino, el empleo de agentes uterotónicos (oxitocina, prostaglandinas), ligadura de arterias uterinas/ hipogástricas y, como último recurso, la histerectomía posparto.

Los casos con diagnóstico de atonía uterina fueron identificados en el libro de reporte de operaciones obstétricas de la sala de operaciones del Hospital San Bartolomé.

Se revisó la historia clínica de cada uno de estos casos identificados y las variables maternas y perinatales fueron ingresadas a una base de datos, empleando el programa estadístico SPSS versión 10. Se incluyó en el análisis el número de unidades de sangre transfundidas a las pacientes, la realización de histerectomía posparto, el estimado subjetivo del volumen de sangre perdida, el hematócrito preparto, el hematócrito posparto y los días de internamiento posparto. 


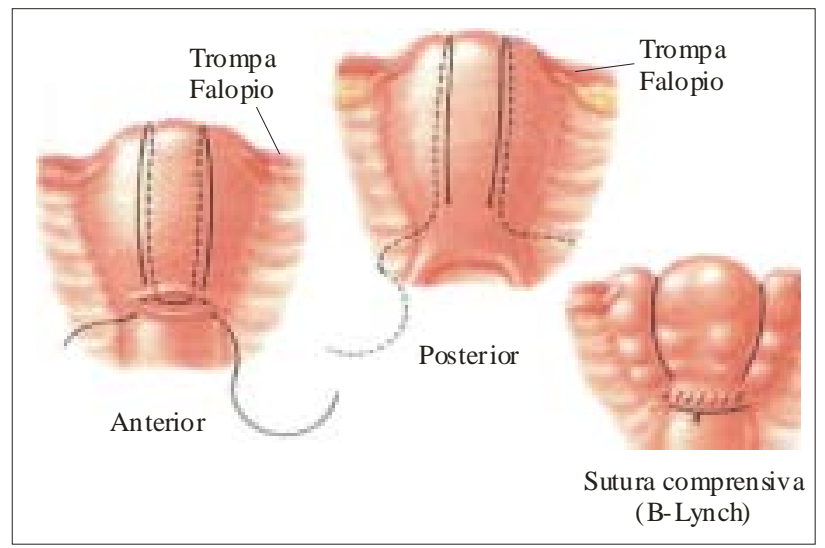

Figura 1. Sutura compresiva del útero (B-Lynch).

EI volumen de la pérdida sanguínea ocurrida en cada uno de los casos fue determinado por el cirujano en forma visual y registrada en la historia clínica.

\section{RESULTADOS}

Durante el periodo de estudio, se identificó 60 casos de hemorragia puerperal por atonía uterina durante la cesárea y se logró recuperar $46(76,6 \%)$ casos de hemorragia puerperal con historia clínica evaluable. E n 30 casos se empleó la técnica de B-Lynch y en 16 el método estándar. L a Tabla 1 muestra las características maternas de ambos grupos. Con excepción del antecedente de abortos previos, no hubo diferencias en las características maternas.

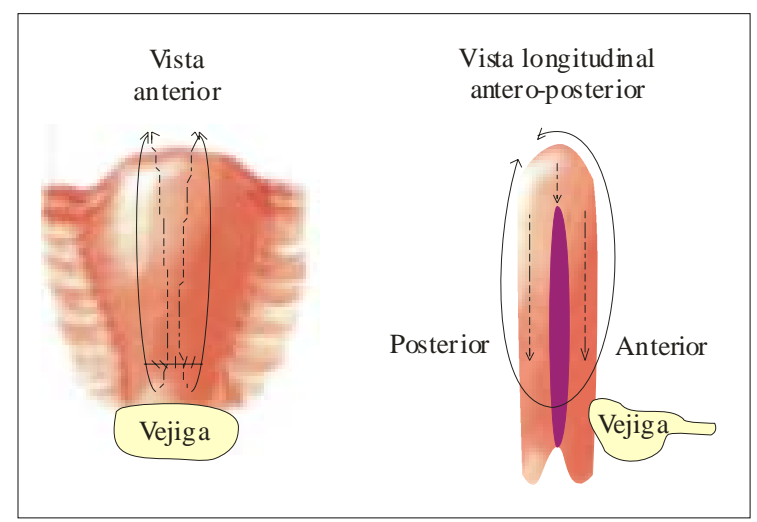

Figura 2. Fundamento de la sutura compresiva del útero.
$\mathrm{L}$ a Tabla 2 muestra el resultado materno. $\mathrm{L}$ a sutura de $B-L$ ynch redujo significativamente el riesgo de histerectomía posparto comparado con el método estándar (OR 0,08, IC 95\% 0,01-0,45, $p<0,01)$. El hematócrito preparto/ posparto y los días de internamiento posparto de los dos grupos de pacientes tratados no fueron estadísticamente diferentes.

Tabla 1 . Características maternas de las pacientes con atonía uterina.

\begin{tabular}{|c|c|c|c|}
\hline Variable & $\begin{array}{c}\text { Sutura } \\
\text { B-Lynch } \\
(x \pm D E)\end{array}$ & $\begin{array}{l}\text { Método } \\
\text { estándar } \\
(x \pm D E)\end{array}$ & $\begin{array}{l}\text { Valor } \\
\text { de } p\end{array}$ \\
\hline Número de casos & 30 & 16 & \\
\hline Edad (años) & $24,9 \pm 5,2$ & $22,8 \pm 7,7$ & N.S. \\
\hline $\begin{array}{l}\text { Número de } \\
\text { abortos previos }\end{array}$ & $0,2 \pm 0,4$ & $0,0 \pm 0,0$ & $<0,05$ \\
\hline $\begin{array}{l}\text { Número de } \\
\text { cesáreas previas }\end{array}$ & $0,1 \pm 0,3$ & $0,3 \pm 0,5$ & N.S. \\
\hline $\begin{array}{l}\text { Número de } \\
\text { controles prenatales }\end{array}$ & $5,5 \pm$ & $6,3 \pm 4,3$ & N.S. \\
\hline Talla $(\mathrm{cm})$ & $153 \pm 3,9$ & $154 \pm 1,4$ & N.S. \\
\hline Peso pregestacional $(\mathrm{kg})$ & $56,8 \pm 7,6$ & $58,3 \pm 12,1$ & N.S. \\
\hline Ganancia de peso $(\mathrm{kg})$ & $11,9 \pm 4,6$ & $16 \pm 7,1$ & N.S. \\
\hline $\begin{array}{l}\text { Edad gestacional } \\
\text { del embarazo }\end{array}$ & $37,4 \pm 3$ & $38,5 \pm 3,4$ & N.S. \\
\hline
\end{tabular}

Tabla 2. Resultado perinatal de las mujeres con atonía uterina.

\begin{tabular}{llll}
\hline \multicolumn{1}{c}{ Variable } & \multicolumn{1}{c}{$\begin{array}{c}\text { Sutura } \\
\text { B-Lynch } \\
(\mathrm{x} \pm \mathrm{DE})\end{array}$} & $\begin{array}{l}\text { Método } \\
\text { estándar } \\
(\mathrm{x} \pm \mathrm{DE})\end{array}$ & $\begin{array}{l}\text { Valor } \\
\text { de } p\end{array}$ \\
\hline $\begin{array}{l}\text { Número de casos } \\
\begin{array}{l}\text { Unidad de sangre } \\
\text { transfundidas }\end{array}\end{array}$ & $0,4 \pm 0,7$ & $2,9 \pm 3,6$ & $<0,05$ \\
$\begin{array}{l}\text { Volumen de } \\
\text { sangre perdida }\end{array}$ & $1144,4 \pm 311,7$ & $1666,9 \pm 794,4$ & $<0,05$ \\
$\begin{array}{l}\text { estimada (mL) } \\
\begin{array}{l}\text { Número de casos } \\
\text { de histerectomía } \\
\text { posparto }\end{array}\end{array}$ & 2 & 8 & \\
$\begin{array}{l}\text { Hematócrito } \\
\text { prenatal (\%) }\end{array}$ & $32,6 \pm 3,6$ & $31,3 \pm 2,9$ & N.S. \\
$\begin{array}{l}\text { Hematócrito } \\
\text { posnatal (\%) }\end{array}$ & $26,6 \pm 6,7$ & $23,3 \pm 4,0$ & N.S. \\
$\begin{array}{l}\text { Peso del neonato (gr) } \\
\text { Talla neonato (cm) }\end{array}$ & $47,8 \pm 4,1 \pm 884,8$ & $3443,3 \pm 329,6$ N.S. \\
$\begin{array}{l}\text { Internamiento } \\
\text { posparto (días) }\end{array}$ & $4,1 \pm 1,1$ & $6,3 \pm 5,7$ & N.S. \\
\hline
\end{tabular}




\section{DISCUSIÓN}

Este estudio demuestra que la sutura compresiva del útero disminuye el volumen de pérdida sanguínea, el empleo de transfusiones sanguíneas y la necesidad de efectuar una histerectomía para controlar la hemorragia puerperal, comparado con el método estándar.

La pérdida sanguínea fue estimada por el médico tratante en forma visual y registrada en la historia clínica. Se acepta que cuando la pérdida sanguínea es mayor de $600 \mathrm{~mL}$ la cantidad de sangrado suele ser subestimada $\left({ }^{17}\right)$. Nosotros asumimos que el error sistemático en la subestimación del sangrado fue similar en ambos grupos; ya que la misma escala de estimación visual fue realizada por los médicos tratantes de los casos y los controles. Por lo que consideramos que la sutura compresiva del útero disminuye significativamente el sangrado vaginal en los casos tratados comparado con los controles.

Los dos primeros casos donde fracasó la técnica de $B-L$ ynch fue debido a que no se tenía mucha experiencia en su empleo y era usada como último recurso después de una hemorragia masiva. A ctualmente recomendamos que el método sea empleado como recurso de primera línea, incluso en lugar de la inyección local de oxitocina en los cuernos uterinos, para evitar la transfusión de hemoderivados. El empleo precoz de la sutura compresiva del útero en casos de atonía uterina durante la cesárea es un factor adicional que disminuye el sangrado puerperal Intraoperatorio en forma importante.

Debido a la característica retrospectiva del estudio, hemos considerado sólo los días de internamiento de las pacientes, el hematócrito posparto y el número de reintervenciones quirúrgicas posparto (histerectomía posparto). Estamos actualmente realizando un estudio de seguimiento de las pacientes que fueron sometidas a la técnica de B-L ynch y que será motivo de una futura publicación.

En conclusión, la sutura compresiva del útero puerperal reduce el sangrado vaginal, la transfusión sanguínea y el empleo de la histerectomía comparado con el método estándar. Por tal motivo, se recomienda su empleo en los casos de hemorragia puerperal debida a atonía uterina.

\section{REFERENCIAS BIBLIOGRÁFICAS}

1. Drife J. M anagement of primary postpartum hemorrhage. Br J Obstet Gynaecol. 1997; 104:275-7.

2. ENDES 2000. Encuesta Demográfica y de Salud Familiar 2000. INEI, USAID, UNICEF, M easure/DHS+. República del Perú. Lima: M acro International Inc.; 2001: 125-36.

3. M inisterio de Salud. Programa N acional de Salud M aterna y Perinatal. Plan Nacional de A cción para la Reducción de la Mortalidad M aterna y Perinatal 1998-2001. Lima: M inisterio de Salud; 2002.

4. Távara L, Sacsa D, Frisancho O, U rquizo R, Carrasco N, Tavera M. Estado actual de la mortalidad materna en los hospitales del Perú. Ginecol O bstet (Perú). 1999;45:38-42.

5. Departamento de Ginecología y Obstetricia, UNM SM . Epidemiología Perinatal 1992-1999. Lima: Hospital Nacional Docente M adre-N iño; 2002.

7. B-L ynch C, Coker A, Lawal A II, A bu J, Cowen M C. The $B-L$ ynch surgical technique for the control of massive postpartum haemorrhage: an alternative to hysterectomy? Five cases reported. Br J Obstet Gynaecol. 1997; 104:372-5.

8. Holtsema H, N ijland R, Huisman A, D ony J, van den Berg PP. The B-L ynch technique for postpartum haemorrhage: an option for every gynaecologist. Eur J Obstet Gynecol Reprod Biol. 2004;115(1):39-42.

9. Pal M, Biswas AK, Bhattacharya SM. B-L ynch brace suturing in primary post-partum hemorrhage during cesarean section. J Obstet Gynaecol Res. 2003;29(5):317-20.

10. M azhar SB, Y asmin S, Gulzar S. M anagement of massive postpartum hemorrhage by "B-L ynch" brace suture. J Coll Physicians Surg Pak. 2003; 13(1):51-2.

11. Smith KL, Baskett TF. U terine compression sutures as an alternative to hysterectomy for severe postpartum hemorrhage. J Obstet Gynaecol Can. 2003;25(3):197-200.

12. Danso D, Reginald P. Combined B-Lynch suture with intrauterine balloon catheter triumphs over massive postpartum haemorrhage. BJ OG. 2002;109(8):963.

13. Hayman RG, A rulkumaran S, Steer PJ. U terine compression sutures: surgical management of postpartum hemorrhage. Obstet Gynecol. 2002;99(3):502-6.

14. Ferguson JE, B ourgeois FJ, U nderwood PB.B-L ynch suture for postpartum hemorrhage. O bstet Gynecol. 2000; 95(6 Pt 2): 1020-2.

15. Santiváñez A. Técnica de B-L ynch para el control de la hemorragia puerperal masiva: Reporte de seis casos en el Hospital San Bartolomé. Gynaecia. 2000;1(1):25-6.

16. Santiváñez A. Técnica de B-Lynch para el control de la hemorragia puerperal masiva. T ema libre presentado al XIII Congreso Peruano de Obstetricia y Ginecología; 20005 al 12 de noviembre. L ima: Sociedad Peruana de Ginecología y Obstetricia; 2000.

17. Duthie SJ, Ghosh A, NgA, Ho PC. Intra-operative blood loss during elective lower segment caesarean section. $\mathrm{Br}$ J Obstet Gynaecol. 1992;99(5):364-7.

Correspondencia:

Dr. Percy Pacora Portella

Departamento de Gineco-O bstetricia - U NM SM

H ospital Nacional D ocente Madre-Niño "San Bartolomé"

Av. Alfonso U garte 825. Lima 1, Perú

Correo-e: percypacora@terra.com.pe 